\title{
1,3-二羟基呫吨酮类化合物的简洁合成及清除亚硝酸根作用研究
}

\author{
毛双双 ${ }^{a}$ 郑欢欢 ${ }^{a}$ 罗翠婷 ${ }^{a}$ 陈河如*,a,b,c \\ ( ${ }^{a}$ 暨南大学药学院中药及天然药物研究所 广州 510632) \\ $\left({ }^{b}\right.$ 中国科学院上海药物研究所新药研究国家重点实验室 上海 201203) \\ ( ${ }^{c}$ 广东省中药药效物质基础及创新药物研究重点实验室 广州 510632)
}

\begin{abstract}
摘要 以间苯三酚为起始原料, 在伊顿试剂的催化作用下，与不同取代水杨酸反应合成 1,3-二美基呫吨酮类化合物 3a $\sim 3112$ 个, 收率 $63 \% \sim 87 \%$, 并探讨了反应机理及结构一反应活性关系. 利用紫外分光光度法测定了所合成化合物对 亚硝酸盐的清除作用, 结果表明, 在 $37{ }^{\circ} \mathrm{C} 、 \mathrm{pH} 3$ 及反应时间为 $30 \mathrm{~min}$ 条件下, 1,3-呫吨酮类化合物对亚硝酸根均有较 好的清除作用，其中化合物 $3 \mathbf{f}$ 和 $3 \mathbf{b}$ 的清除作用最强，清除率达 34\%. 同时对 1,3-二差基呫吨酮类化合物清除亚硝酸盐 的作用机制及构效关系进行了探讨.
\end{abstract}

关键词＼cjkstart呫吨酮；有机合成；亚硝酸盐；癌症防治；伊顿试剂

\section{Concise Syntheses of 1,3-Dihydroxylxanthones and Their Clearance Effections against Nitrite}

\author{
Mao, Shuangshuang ${ }^{a} \quad$ Zheng, Huanhuan $^{a} \quad$ Luo, Cuiting $^{a}$ Chen, Heru ${ }^{*, a, b, c}$ \\ ( ${ }^{a}$ Institute of Traditional Chinese Medicine and Natural Products, College of Pharmacy, \\ Jinan University, Guangzhou 510632) \\ ( ${ }^{b}$ State Key Laboratory of Drug Research, Shanghai Institute of Materia Medica, Chinese Academy of Sciences, \\ Shanghai 201203) \\ ( ${ }^{c}$ Guangdong Province Key Laboratory of Pharmacodynamic Constituents of TCM and New Drugs Research, \\ Jinan University, Guangzhou 510632)
}

\begin{abstract}
Twelve 1,3-dihydroxylxanthones (3a $\sim 31)$ were synthesized under the catalysis of Eaton's reagent applying phloroglucinol and substituted salicylic acids as starting materials. The yields were from $63 \%$ to $87 \%$. The reaction mechanism and their structure-reaction activity relationship have been discussed. The clearance effections of all the prepared xanthones against nitrite have been investigated by UV spectrophotometry. It is disclosed that all the tested xanthones have obvious clearance effections against nitrite at the conditions of $\mathrm{pH} 3$, room temperature, and 30 min treatment. 3b and $\mathbf{3 f}$ are identified as the most active compounds with clearance rate up to $34.0 \%$. The clearance mechanism of xanthones against nitrite and their structure-activity relationships were also included here.
\end{abstract}

Keywords xanthones; organic synthesis; nitrite; cancer prevention; Eaton's reagent

亚硝酸盐在食品安全中所引起的问题早已受到关 注. 亚硝酸盐广泛存在于土壤、水体中，在食品加工及 储存过程中也有亚硝酸盐的产生. 亚硝酸盐被人体吸收 后，可与人体中含氨基的功能分子包括蛋白质、DNA 及 信号分子反应生成 $N$-亚硝基化合物 (nitrosodimethylamin, 简称 NDMA), 该化合物是目前所知的最强的化
学致癌物质之一，能引起人和动物的胃、肝脏等多种器 官的恶性慢性肿瘤 ${ }^{[1]}$. 亚硝酸盐无论在实验室和自然条 件下还是在人和动物体内均能反应生成 NDMA, 尤其 在人和动物的胃中更适于生成 $\mathrm{NDMA}^{[2]}$. 从亚硝胺生 成的动力学来看, 其生成速度与亚硝酸根 $\left(\mathrm{NO}_{2}^{-}\right)$的平 方成正比 ${ }^{[3]}$. 因此, 阻断 NDMA 的合成或清除亚硝胺的

* E-mail: thrchen@jnu.edu.cn

Received October 11, 2013; revised November 10, 2013; published online November 21, 2013.

Project supported by the National Natural Science Foundation of China (No. 81172982), the Guangdong Provincial Project of Science and Technology (No. 2010A030100006) and the State Key Laboratory of Drug Research (No. SIMM1302KF-12).

国家自然科学基金(No. 81172982)、广东省科技计划社会发展重点(No. 2010A030100006)、新药研究国家重点实验室开放基金(No. SIMM1302KF-12)资助 项目. 
前体化合物一一亚硝酸根是预防癌症的有效途径之一.

薛长晖 ${ }^{[2]}$ 曾报道特色藏药藏茵陈的乙醇提取液对亚 硝酸盐具有良好的清除作用. 作为藏茵陈的主要药效成 分之一, 呫吨酮类化合物是否具有清除亚硝酸盐的作 用? 这是本文所关注的科学问题, 此方面的工作未见文 献报道. 众所周知, 呫吨酮(xanthones) 是以二苯骈- $\gamma$-吡 喃酮为母核的一类化合物, 主要存在于龙胆科 ${ }^{[4]}$ 和藤黄

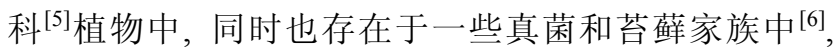
是近代天然产物中的一类重要活性成分 ${ }^{[7]}$. 由于呫吨酮 类化合物的独特结构使得它们能够与不同的药物靶标 作用 ${ }^{[8]}$, 已经报道该类化合物具有抗氧化 ${ }^{[9,10]}$ 、抗过 敏 $^{[11]}$ 、抗菌 ${ }^{[12]}$ 、抗炎 ${ }^{[13]}$ 、抗 HIV-1 病毒 ${ }^{[14]}$ 、降血糖 ${ }^{[15,16]}$ 、 抗肿瘤 ${ }^{[17,18]}$ 、抗㾏疾 ${ }^{[19]}$ 和延缓神经退行性疾病进 程 ${ }^{[14,15,19]}$ 等作用. 基于对呫吨酮类化合物在新药开发上 的潜在价值, 其合成引起了科学家的广泛兴趣. 近年来, 已有多篇文献报道了基于呫吨酮的新药设计合成及其 药理活性研究 ${ }^{[17,18,20 ~ 22]}$.

从呫吨酮的结构上看, 它包含有多酚结构, 理论上 应当具有清除亚硝酸根的作用. 因此, 本文拟采用简 洁、经济的方法合成系列呫吨酮类化合物, 并研究它们 对亚硝酸根的清除作用及其构效关系, 为研发具有预防 和治疗癌症新药奠定科学基础. 目标化合物的合成路线 见 Eq. 1.

\section{1 结果与讨论}

\section{1 呫吨酮的合成}

呫吨酮类化合物的合成有多种方法, 胡利红等 ${ }^{[23]}$ 已经作了很好的综述. 本实验以伊顿试剂(Eaton's reagent 是重量比 $1: 10$ 的 $\left.\mathrm{P}_{2} \mathrm{O}_{5} / \mathrm{CH}_{3} \mathrm{SO}_{3} \mathrm{H}\right)^{[24]}$ 为催化剂兼 溶剂, 以间苯三酚为起始原料，与不同取代的水杨酸类 化合物直接缩合环化，以 $63 \% \sim 87 \%$ 的收率合成系列呫 吨酮类化合物(表 1). 其中，水杨酸自身与间苯三酚的反 应收率最高, 达 $87 \%$ (3d). 通常, 当水杨酸类化合物芳 环上有 $+\mathrm{I}$ 或 $+\mathrm{C}$ 效应的基团时，收率将降低，尤其当这 些基团处于羧基的邻、对位时，效应更显著，如 $\mathbf{3 b}$ 和 $\mathbf{3 a}$, 其反应收率只有 $63 \%$ 和 $75 \%$; 当水杨酸类化合物羟基的 邻、对位有 $-\mathrm{I}$ 或 $-\mathrm{C}$ 效应的基团时，同样会使反应收率 下降，如 $3 \mathrm{~g}$ 和 $3 \mathrm{~h}$ ，反应收率分别为 $74 \%$ 和 $78 \%$. 水杨酸 类化合物的芳环上不能有游离的氨基，氨基必需保护才 能顺利发生缩合环化反应(如 3I). 实验中发现, 当间苯 三酚投入量由 1 equiv. 提高到 1.5 equiv. 时，产率明显提 高. 可见, 过量的间苯三酚有助于促进反应的进行.

本实验条件下，间苯三酚与水杨酸类化合物的缩合 环化机理可能有 3 种(图 1). 其一是水杨酸类化合物在伊 顿试剂的催化作用下与间苯三酚发生 Friedel-Craft 酰化

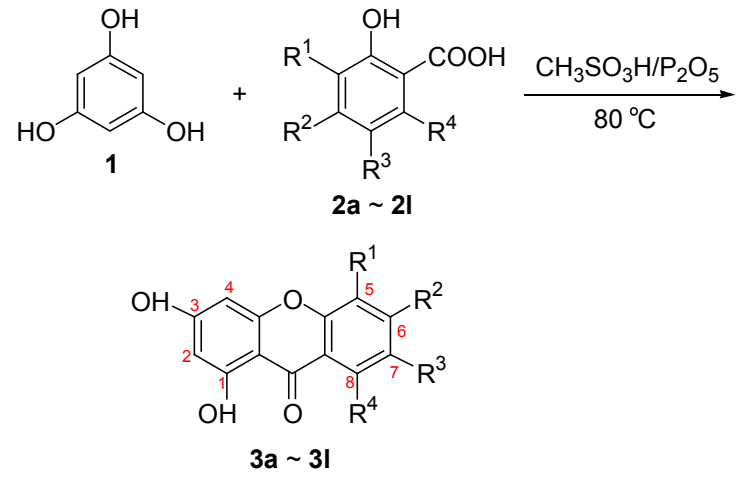

a: $R^{1}=R^{3}=R^{4}=H, R^{2}=O H$

b: $R^{1}=R^{3}=H, R^{2}=R^{4}=O H$

c: $\mathrm{R}^{1}=\mathrm{R}^{2}=\mathrm{R}^{4}=\mathrm{H}, \mathrm{R}^{3}=\mathrm{OMe}$

d: $R^{1}=R^{2}=R^{3}=R^{4}=H$

e: $R^{1}=M e, R^{2}=R^{3}=R^{4}=H$

f: $R^{1}=R^{3}=R^{4}=H, R^{2}=M e$

g: $R^{1}=R^{2}=R^{4}=H, R^{3}=C l$

h: $R^{1}=R^{2}=R^{4}=H, R^{3}=B r$

i: $R^{1}=R^{2}=R^{3}=H, R^{4}=M e$

j: $\mathrm{R}^{1}=\mathrm{R}^{4}=\mathrm{H}, \mathrm{R}^{2} / \mathrm{R}^{3}=\mathrm{Ph}[b]$

k: $R^{1} / R^{2}=P h[b], R^{3}=R^{4}=H$

l: $\mathrm{R}^{1}=\mathrm{R}^{2}=\mathrm{R}^{4}=\mathrm{H}, \mathrm{R}^{3}=\mathrm{NHAC}$

表 1 呫吨酮类化合物的合成产率

Table 1 Synthetic yields of xanthones

\begin{tabular}{cc||cc}
\hline Compd. & Yield/\% & Compd. & Yield/\% \\
\hline 3a & 75 & $\mathbf{3 g}$ & 74 \\
3b & 63 & $\mathbf{3 h}$ & 78 \\
3c & 80 & $\mathbf{3 i}$ & 82 \\
3d & 87 & $\mathbf{3 j}$ & 84 \\
3e & 85 & $\mathbf{3 k}$ & 81 \\
3f & 84 & $\mathbf{3 1}$ & 78 \\
\hline
\end{tabular}

反应得芳酮，然后再发生分子内亲核反应成醚键(机理 I); 其二是水杨酸类化合物先与间苯三酚反应成酯，再 经 Fries 重排得芳酮，最后成醚键环化(机理 II); 第三条 路线与第二条路线相似，先反应生成酯，再经 Smiles 重 排得芳醚，最后经 Friedel-Craft 酰化反应成环(机理 III). 三种机理都有相应的成功实例 ${ }^{[25 ~ 27]}$. 不难看出, 表 1 所 体现出来的结构一反应活性关系与任何一种反应机理的 推论一致。

本实验还尝试了分别以 1,2,4-苯三酚、对苯二酚、 间苯二酚、苯酚替代间苯三酚与水杨酸类化合物反应合 成占吨酮类化合物的可能性. 结果发现，不管是在相同 条件下，或是提高反应温度、延长反应时间，均不能得 到呫吨酮类化合物. 可见，该反应成功的关键是亲核受 体(如间苯三酚)要有足够大的活性. 从结构上看，间苯 三酚有三个强的致活羟基，而且活化方向一致，所以不 管是图 1 中的任何一种机理，它都是活泼的反应底物， 能很好地发生缩合环化反应; 相比而言, 1,2,4-苯三酚虽 然也有三个强的致活羟基，但其致活的方向不完全一 


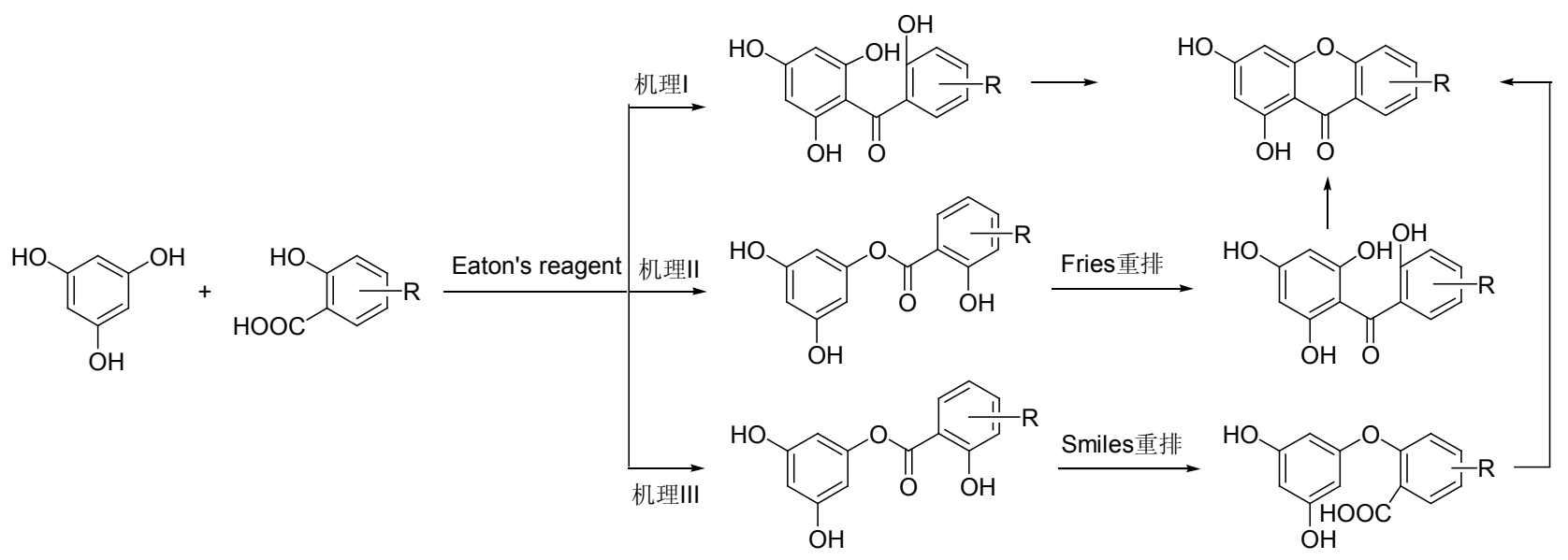

图 1 伊顿试剂催化下合成呫吨酮的三种可能机理

Figure 1 Three possible mechanisms of the syntheses of xanthones catalyzed by Eaton's reagent

致，其每个反应位点都没有间苯三酚活泼，所以无法发 生反应; 其它酚类化合物显然更加不活泼.

以伊顿试剂为催化剂合成呫吨酮类化合物已有文 献报道 ${ }^{[28]}$. 在酚羟基不保护的条件下, 图 1 所示的三种 反应机理都有可能. 本实验曾分离得到对苯二酚分别与 2a 和 $2 \mathbf{j}$ 反应的中间体化合物 w-1 和 $w-2$, 为机理 II 和 III 提供有力的证据. 但机理 I 的中间体化合物较难获得, 因为若能形成芳酮, 则成环得呫吨酮的速度很快, 无法 停留在芳酮阶段.

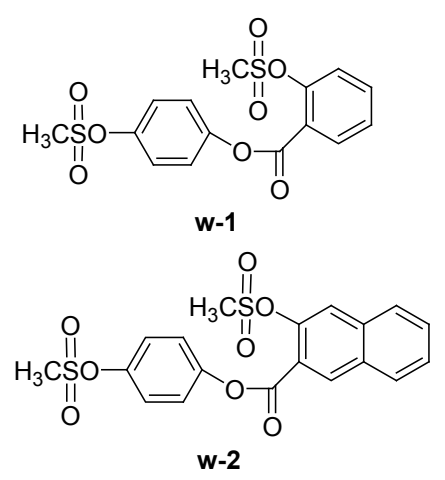

理论上, 伊顿试剂与多聚磷酸(PPA)作用相似. 但 PPA 非常粘稠, 在低于 $60 \sim 90{ }^{\circ} \mathrm{C}$ 温度下很难实现有效 搅拌, 而且大部分的有机化合物在 PPA 中溶解度很小. 相比之下, 伊顿试剂克服了 PPA 的所有缺点, 可以作为 催化剂兼溶剂使用, 而且价格低廉, 后处理方便, 污染 小, 毒性低, 在本实验中是一种高效低毒、经济实用的 缩合环化剂.

\section{$1.2 \mathrm{NO}_{2}^{-}$清除作用}

本实验利用紫外分光光度法测定了在 $37^{\circ} \mathrm{C} 、 \mathrm{pH}$ 值 分别为 3 和 7 两种条件下呫吨酮类化合物 $3 \mathbf{a} \sim 31$ 对 $\mathrm{NO}_{2}^{-}$的清除作用. 结果见表 2. 显然, 化合物 3a $\sim 31$ 对 $\mathrm{NO}_{2}^{-}$均有一定的清除作用. 在 $\mathrm{pH} 3$ 介质中的清除作用
比在 $\mathrm{pH} 7$ 介质中的清除作用要强. 其中, 在 $\mathrm{pH} 3$ 时, 化 合物 $3 \mathbf{b}$ 和 $3 \mathbf{f}$ 对亚硝酸根的清除率最高，达 $34.0 \%$. 其它 化合物如 $\mathbf{3 b}, 3 \mathbf{g}, 3 \mathbf{h}, 3 \mathbf{e}$ 和 $\mathbf{3 j}$ 也有较高的清除率, 均达 $30 \%$ 左右. 对比呫吨酮类化合物的结构, 它们在 1,3 位 上都有酚羟基. 若 5,6,7 位被 $+\mathrm{I}$ 或 $-\mathrm{C}$ 效应的基团如甲 基、卤原子 $(\mathrm{Cl}, \mathrm{Br})$ 取代时，清除作用增强; 若 7,8 位被 $+\mathrm{I}$ 或 $+\mathrm{C}$ 效应的基团取代, 如 $\mathbf{3 c}, \mathbf{3 i}, \mathbf{3 1}$, 其清除作用减 弱. 若只在 6-位有羟基, 如 3a, 则清除作用并没有得到 增强; 若在 6,8-位上同时有羟基的存在，如 $\mathbf{3 b}$, 则其对 $\mathrm{NO}_{2}^{-}$的清除作用大大增强.

表 2 呫吨酮类化合物对 $\mathrm{NO}_{2}^{-}$的清除作用

Table 2 Clearance effections of xanthones against $\mathrm{NO}_{2}^{-}$

\begin{tabular}{ccc}
\hline \multirow{2}{*}{ Compd. } & \multicolumn{2}{c}{ 亚硝酸根清除率/\% } \\
\cline { 2 - 3 } & $\mathrm{pH} \mathrm{3}$ & $\mathrm{pH} \mathrm{7}$ \\
\hline $\mathbf{3 a}$ & 11.5 & 4.9 \\
$\mathbf{3 b}$ & 34.0 & 12.2 \\
$\mathbf{3 c}$ & 9.6 & 3.0 \\
$\mathbf{3 d}$ & 18.0 & a $^{a}$ \\
$\mathbf{3 e}$ & 28.1 & 9.2 \\
$\mathbf{3 f}$ & 34.0 & 19.7 \\
$\mathbf{3 g}$ & 32.9 & 23.8 \\
$\mathbf{3 h}$ & 29.4 & 9.2 \\
$\mathbf{3 i}$ & 12.0 & $-{ }^{a}$ \\
$\mathbf{3 j}$ & 26.0 & 15.6 \\
$\mathbf{3 k}$ & 19.3 & 12.8 \\
$\mathbf{3 1}$ & 16.6 & 0.2 \\
\hline a“一 表示没有数据. & &
\end{tabular}

我们推测, 呫吨酮类化合物清除 $\mathrm{NO}_{2}^{-}$的作用来自 于它们之间能发生亲电取代反应(图 2). 在酸性环境中, 亚硝酸根容易产生活泼的 $\mathrm{NO}^{+}$, 更容易与呫吨酮类化 合物发生亚硝化反应. 所以, 呫吨酮类化合物在 $\mathrm{pH} 3$ 的 介质中具有更好的清除作用; 而在 $\mathrm{pH} 7$ 介质中, 不利于 $\mathrm{NO}^{+}$的产生，其清除作用相对较弱.

本实验中, $\mathrm{pH} 3$ 介质模拟的是人胃液的生理环境, 


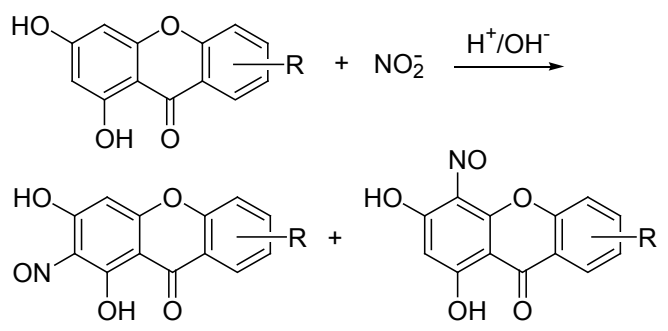

图 2 呫吨酮类化合物清除 $\mathrm{NO}_{2}^{-}$的可能机理

Figure 2 Putative mechanism of the nitrite clearance of xanthones

pH 7 介质模拟的是肠液的生理环境. 据报道 ${ }^{[2]}$, 亚硝酸 盐在人和动物的胃中更适于生成强致癌性物质 NDMA. 显然，若能在胃中有效清除 NDMA，那么其预防癌症的 效果将较佳; 而上述的数据显示, 呫吨酮类化合物在模 拟胃液的环境中对 $\mathrm{NO}_{2}^{-}$的清除作用更为突出, 所以作 者认为呫吨酮类化合物有可能作为预防癌症的候选化 合物.

\section{2 结论}

本实验利用伊顿试剂作催化剂兼溶剂，成功合成了 12 个 1,3-二羟基呫吨酮类化合物，收率 63\% 87\%, 并 探讨了反应机理及结构-反应活性关系, 同时研究了所 合成化合物对亚硝酸根的清除作用. 实验结果表明, 1,3-二美基呫吨酮类化合物在 $\mathrm{pH} 3$ 介质中对 $\mathrm{NO}_{2}^{-}$有较 好的清除作用, 以化合物 $\mathbf{3 b}$ 和 $\mathbf{3 f}$ 对 $\mathrm{NO}_{2}^{-}$的清除率最高, 达 $34.0 \%$; 在 $\mathrm{pH} 7$ 介质中的清除作用相对较弱, 其作用 强度的差异与其机制有关. 即在酸性环境, 亚硝酸根更 容易产生活泼的 $\mathrm{NO}^{+}$与 1,3 -二羟基呫吨酮类化合物发生 反应. 取代基随其电子效应及所在位置的不同, 对清除 能力产生不同的影响. 当 $+\mathrm{I}$ 或 $-\mathrm{C}$ 效应的基团如甲基、 卤原子 $(\mathrm{Cl}, \mathrm{Br})$ 位于 $5 \sim 7$ 位取代时, 清除作用增强; 当 $+\mathrm{I}$ 或 $+\mathrm{C}$ 效应的基团处于 7,8 位时, 其清除作用减弱.

\section{3 实验部分}

\section{1 仪器与试剂}

Bruker-AV300MHz 型核磁共振仪(瑞士 Bruker 公 司); DHG-9140A 型恒温干燥箱(上海浦东荣丰科学仪器 有限公司); CLJBQ-3 型恒温磁力搅拌器(郑州长城科工 贸有限公司); EYELA 型旋转蒸发仪(东京理化器械独资 工厂); LGJ-12 冷冻干燥机(巩义市英峪予华仪器有限公 司); FA2104 型电子天平(上海恒平科学仪器有限公司); TU-1810S 紫外分光光度计(北京普析通仪器有限公司); LC-UV100 液相色谱仪(上海伍丰科学仪器有限公司); DZF-150 型小型真空干燥箱(郑州长城科工贸有限公司); KQ3200E 型超声波清洗器(昆山市超声仪器有限公司).

五氧化二磷(AR, 天津百世试剂); 无水硫酸镁(AR,
天津百世试剂); 硅胶(200 300 目, 烟台化学工业研究 所); 醋酸䣶( $\mathrm{AR}$, 广州化学试剂厂); 甲醇( $\mathrm{GR}$, 安徽时 联特种溶剂有限公司); 甲磺酸、间苯三酚、水杨酸、盐 酸萗乙二胺、对甲基苯磺酸、亚硝酸钠、柠檬酸、磷酸 氢二钠等均为 aladdin 试剂(上海晶纯科技有限公司).

\section{2 实验方法}

\section{2 .1 化合物的合成}

5-乙酰氨基-2-羊基苯甲酸(2I)的合成: 称取 5-氨基 水杨酸 $765 \mathrm{mg}(5.0 \mathrm{~mol})$ 于 $50 \mathrm{~mL}$ 圆底烧瓶中, 用注射器 量取 $0.5 \mathrm{~mL}$ (约 $5.0 \mathrm{~mol}$ )乙酸䣶加入到反应瓶中, 用适 量重蒸过的甲醇做溶剂, 于 $65{ }^{\circ} \mathrm{C}$ 回流 $8 \mathrm{~h}$. 室温下旋转 蒸除溶剂, 残留物用乙酸乙酯萃取四次, 每次 $20 \mathrm{~mL}$, 合并有机相, 饱和 $\mathrm{NaCl}$ 洗涤 $(20 \mathrm{~mL} \times 3)$, 无水 $\mathrm{Na}_{2} \mathrm{SO}_{4}$ 干燥, 旋蒸除去溶剂. 残留物用 RP-HPLC 分离纯化 (27\%的甲醇水为洗脱剂), 得浅黄色固体 $781 \mathrm{mg}$, 收率 $80 \%$. ${ }^{1} \mathrm{H}$ NMR (DMSO- $\left.d_{6}, 300 \mathrm{MHz}\right) \delta: 9.89(\mathrm{~s}, 1 \mathrm{H}, \mathrm{OH}$ ), 8.09 (d, $J=2.5 \mathrm{~Hz}, 1 \mathrm{H}, \mathrm{H}-6), 7.64$ (dd, $J=8.9,2.6 \mathrm{~Hz}, 1 \mathrm{H}$, $\mathrm{H}-4), 6.90$ (d, $J=8.9 \mathrm{~Hz}, 1 \mathrm{H}, \mathrm{H}-3), 2.01$ (s, 3H, $\mathrm{COCH}_{3}$ ); MS-ESI $m / z: 196.1[\mathrm{M}+\mathrm{H}]^{+}$.

呫吨酮类化合物 $\mathbf{3 a} \sim \mathbf{3 k}$ 的合成通法: 根据文献[24] 制备 Eaton's Reagent. 将 $6 \mathrm{~g}$ 重蒸过的甲磺酸加入到 25 $\mathrm{mL}$ 圆底烧瓶中, 再加入 $0.6 \mathrm{~g}$ 的 $\mathrm{P}_{2} \mathrm{O}_{5}, 80{ }^{\circ} \mathrm{C}$ 条件下摚拌 $1 \mathrm{~h}$, 得到淡黄色的溶剂备用. 参照文献[28]合成呫吨酮 类化合物 3a $\sim 3 \mathbf{k}$. 将间苯三酚 $97 \mathrm{mg}(0.6 \mathrm{mmol})$ 和取 代水杨酸类化合物 $(0.5 \mathrm{~mol})$ 加入到 $25 \mathrm{~mL}$ 圆底烧瓶 中，随后在摚拌下缓慢滴加新制备的 Eaton's Reagent, 升温至 $80{ }^{\circ} \mathrm{C}$ 反应 $1 \mathrm{~h}$. 反应结束后, 将反应混合物冷却 至室温, 倒入冰水中剧烈搅拌 $2 \mathrm{~h}$, 有黄色粉末析出, 过 滤, 固体用蒸馏水清洗数次直至滤液的 $\mathrm{pH}$ 值接近 6 , 收 集滤饼，用 RP-HPLC 分离纯化(洗脱剂为 $65 \%$ 的甲醇水 加 $0.1 \%$ 的甲酸), 旋蒸去除甲醇, 冷冻干燥得黄色粉末.

1,3,6-三羟基呫吨酮 (3a): 黄色粉末，产率 78.0\%. ${ }^{1} \mathrm{H}$ NMR (DMSO- $\left.d_{6}, 300 \mathrm{MHz}\right) \delta: 13.05$ (s, $\left.1 \mathrm{H}, \mathrm{OH}-1\right)$, 11.03 (s, 1H, OH-6), 10.95 (s, 1H, OH-3), 7.95 (d, $J=9.0$ $\mathrm{Hz}, 1 \mathrm{H}, \mathrm{H}-8), 6.88$ (dd, $J=2.3,8.9 \mathrm{~Hz}, 1 \mathrm{H}, \mathrm{H}-7), 6.80$ (d, $J=2.4 \mathrm{~Hz}, 1 \mathrm{H}, \mathrm{H}-5), 6.33$ (d, $J=3.0 \mathrm{~Hz}, 1 \mathrm{H}, \mathrm{H}-2), 6.16$ (d, $J=3.0 \mathrm{~Hz}, 1 \mathrm{H}, \mathrm{H}-4)$ ) ${ }^{13} \mathrm{C}$ NMR (DMSO- $\left.d_{6}, 75 \mathrm{MHz}\right) \delta$ : 179.5 (C-9), 165.6 (C-3), 164.7 (C-6), 163.3 (C-1), 157.9 (C-4a), 157.8 (C-4b), 127.6 (C-8), 114.4 (C-8a), 112.8 (C-7), 102.5 (C-8b), 102.1 (C-5), 98.4 (C-2), 94.4 (C-4); MS-ESI $m / z: 245.3[\mathrm{M}+\mathrm{H}]^{+}$. 表征数据和理化性质与文 献[28, 29]一致.

1,3,6,8-四羟基呫吨酩 $(3 \mathbf{b})$ ：淡红色粉末，产率 $63 \%$. UV-Vis $\left(\mathrm{CH}_{3} \mathrm{COCH}_{3}\right) \lambda_{\text {max }}: 330,368 \mathrm{~nm} ;{ }^{1} \mathrm{H}$ NMR $\left(\mathrm{CD}_{3} \mathrm{OD}, 300 \mathrm{MHz}\right) \delta: 6.23(\mathrm{~d}, J=2.3 \mathrm{~Hz}, 2 \mathrm{H}, \mathrm{H}-4, \mathrm{H}-5)$, 
$6.12(\mathrm{~d}, J=3.0 \mathrm{~Hz}, 2 \mathrm{H}, \mathrm{H}-2, \mathrm{H}-7) ;{ }^{13} \mathrm{C}$ NMR (DMSO- $d_{6}$, $300 \mathrm{MHz}$ ) $\delta$ : 184.2 (C-9), 167.1 (2C, C-3/C-6), 164.0 (2C, C-4a/C-4b), 159.1 (2C, C-1/C-8), 102.1 (2C, C-8a/C-8b), 99.3 (2C, C-2/C-7), 95.2 (2C, C-4/C-5); IR (KBr) v: 3377, $1657,1617,1586,1487,1320,1161,820 \mathrm{~cm}^{-1}$; MS-ESI $m / z: 261.3[\mathrm{M}+\mathrm{H}]^{+}$; HRMS calcd for $\mathrm{C}_{13} \mathrm{H}_{8} \mathrm{O}_{6} \quad 260.0314$, found 260.0321 .

1,3-二羟基-7-甲氧基呫吨酮(3c)：黄色粉末，产率 80\%. ${ }^{1} \mathrm{H}$ NMR (DMSO-d $\left.6,300 \mathrm{MHz}\right) \delta$ : 12.81 (s, 1H, OH-1), 11.21 (s, 1H, OH-3), 7.50 (d, $J=8.7$ Hz, 1H, H-5), 7.43 (s, 1H, H-8), 7.38 (d, J=9.0 Hz, 1H, H-6), 6.36 (s, $1 \mathrm{H}, \mathrm{H}-4), 6.19$ (s, 1H, H-2), 3.84 (s, 3H, $\left.\mathrm{OCH}_{3}\right) ;{ }^{13} \mathrm{C} \mathrm{NMR}$ (DMSO- $d_{6}, 75$ MHz) $\delta: 179.9$ (C-9), 166.3 (C-3), 163.2 (C-1), 157.8 (C-4a), 156.1 (C-7), 150.4 (C-4b), 124.8 (C-6), 120.6 (C-8a), 119.6 (C-5), 105.7 (C-8), 102.4 (C-8b), 98.5 (C-2), 94.3 (C-4), $56.2\left(\mathrm{OCH}_{3}\right)$; MS-ESI $m / z$ : $259.2[\mathrm{M}+\mathrm{H}]^{+}$. 表征数据和理化性质与参考文献 $[30]$ 一 致.

1,3-二羟基呫吨酩(3d): 黄色粉末，产率 $87 \%$. ${ }^{1} \mathrm{H}$ $\mathrm{NMR}\left(\mathrm{CD}_{3} \mathrm{OD}, 300 \mathrm{MHz}\right) \delta$ : $8.18(\mathrm{dd}, J=1.4,7.9 \mathrm{~Hz}, 1 \mathrm{H}$, H-8), $7.89 \sim 7.69$ (m, 1H, H-6), 7.48 (d, $J=8.0 \mathrm{~Hz}, 1 \mathrm{H}$, H-5), $7.44 \sim 7.37$ (m, 1H, H-7), 6.36 (d, J=1.9 Hz, 1H, $\mathrm{H}-2), 6.20$ (d, $J=1.9 \mathrm{~Hz}, 1 \mathrm{H}, \mathrm{H}-4) ;{ }^{13} \mathrm{C} \mathrm{NMR}\left(\mathrm{CD}_{3} \mathrm{OD}\right.$, $300 \mathrm{MHz}) \delta$ : 180.4 (C-9), 166.0 (C-3), 163.4 (C-1), 158.0 (C-4a), 156.0 (C-4b), 134.9 (C-6), 125.1 (C-8), 123.8 (C-8a), 120.2 (C-7), 117.3 (C-5), 102.5 (C-8b), 97.8 (C-4), 93.7 (C-2); MS-ESI $m / z: 229.2[\mathrm{M}+\mathrm{H}]^{+}$. 表征数据和理 化性质与参考文献[31]一致.

1,3-二羟基-5-甲基呫吨酮(3e): 黄色粉末，产率 85\%. ${ }^{1} \mathrm{H}$ NMR (DMSO-d $\left.6,300 \mathrm{MHz}\right) \delta$ : 12.84 (s, 1H, OH-1), 11.06 (s, 1H, OH-3), 7.93 (d, $J=7.2 \mathrm{~Hz}, 1 \mathrm{H}, \mathrm{H}-8$ ), 7.68 (d, $J=7.2 \mathrm{~Hz}, 1 \mathrm{H}, \mathrm{H}-6), 7.33$ (dd, $J=7.2,7.2 \mathrm{~Hz}, 1 \mathrm{H}$, H-7), 6.40 (d, $J=1.8,1 \mathrm{H}, \mathrm{H}-4), 6.20$ (d, $J=1.8 \mathrm{~Hz}, 1 \mathrm{H}$, $\mathrm{H}-2), 2.46$ (s, $\left.3 \mathrm{H}, \mathrm{CH}_{3}\right) ;{ }^{13} \mathrm{C}$ NMR (DMSO- $d_{6}, 300 \mathrm{MHz}$ ) $\delta$ : 180.0 (C-9), 165.8 (C-3), 162.8 (C-1), 157.2 (C-4a), 153.6 (C-4b), 136.2 (C-6), 126.6 (C-8), 122.8 (C-8a), 119.6 (C-5), 102.0 (C-8b), 98.1 (C-4), 94.1 (C-2); MS-ESI $m / z: 243.3[\mathrm{M}+\mathrm{H}]^{+}$. 表征数据和理化性质与参考文献 [32]一致.

1,3-二羟基-6-甲基呫吨酮(3f)：黄色粉末，产率 84\%. ${ }^{1} \mathrm{H}$ NMR (DMSO-d, $\left.300 \mathrm{MHz}\right) \delta: 12.87$ (s, 1H, OH-1), 11.05 (s, 1H, OH-3), 7.98 (d, $J=8.1 \mathrm{~Hz}, 1 \mathrm{H}, \mathrm{H}-8$ ), 7.37 (s, 1H, H-5), 7.26 (d, J=8.1 Hz, 1H, H-7), 6.36 (d, $J=2.1 \mathrm{~Hz}, 1 \mathrm{H}, \mathrm{H}-4), 6.19$ (d, $J=2.1 \mathrm{~Hz}, 1 \mathrm{H}, \mathrm{H}-3), 2.45$ (s, $\left.3 \mathrm{H}, \mathrm{CH}_{3}\right) ;{ }^{13} \mathrm{C}$ NMR (DMSO- $\left.d_{6}, 75 \mathrm{MHz}\right) \delta$ : 180.1 (C-9),
166.2 (C-3), 163.3 (C-1), 157.8 (C-4a), 155.9 (C-4b), 147.4 (C-6), 126.2 (C-8), 125.5 (C-8a), 118.0 (C-7), 117.8 (C-5), 102.5 (C-8b), 98.5 (C-4), 94.5 (C-2), $21.9\left(\mathrm{CH}_{3}\right)$; MS-ESI $m / z: 243.4[\mathrm{M}+\mathrm{H}]^{+}$. 表征数据和理化性质与参 考文献[32]一致.

1,3-二羟基-7-氯呫吨酮(3g)：黄色粉末，产率 74\%. ${ }^{1} \mathrm{H}$ NMR (DMSO-d $\left.d_{6}, 300 \mathrm{MHz}\right) \delta$ : 12.55 (s, 1H, OH-1), $11.21(\mathrm{~s}, 1 \mathrm{H}, \mathrm{OH}-3), 8.00$ (d, $J=2.6 \mathrm{~Hz}, 1 \mathrm{H}, \mathrm{H}-8), 7.86$ (dd, $J=8.9,2.6 \mathrm{~Hz}, 1 \mathrm{H}, \mathrm{H}-6), 7.62$ (d, $J=8.9 \mathrm{~Hz}, 1 \mathrm{H}$, H-5), 6.38 (d, $J=2.0 \mathrm{~Hz}, 1 \mathrm{H}, \mathrm{H}-4), 6.22$ (d, $J=2.0 \mathrm{~Hz}$, $1 \mathrm{H}, \mathrm{H}-2) ;{ }^{13} \mathrm{C}$ NMR (DMSO- $\left.d_{6}, 75 \mathrm{MHz}\right) \delta$ : 179.0 (C-9), 166.7 (C-3), 163.2 (C-1), 157.7 (C-4a), 154.4 (C-4b), 135.7 (C-6), 129.1 (C-8), 124.6 (C-8a), 121.5 (C-7), 120.7 (C-5), 102.6 (C-8b), 98.8 (C-4), 94.7 (C-2); MS-ESI m/z: $263.3[\mathrm{M}+\mathrm{H}]^{+}$. 表征数据和理化性质与参考文献[33] 致.

1,3-二羟基-7-溴呫吨酮 (3h): 黄色粉末, 产率 $78 \%$. ${ }^{1} \mathrm{H}$ NMR (DMSO- $\left.d_{6}, 300 \mathrm{MHz}\right) \delta: 12.55$ (s, 1H, OH-1), 11.22 (s, 1H, OH-3), 8.13 (d, $J=2.4 \mathrm{~Hz}, 1 \mathrm{H}, \mathrm{H}-8)$, 7.97 (dd, $J=8.9,2.6 \mathrm{~Hz}, 1 \mathrm{H}, \mathrm{H}-6), 7.55$ (d, $J=8.9 \mathrm{~Hz}, 1 \mathrm{H}$, H-5), 6.38 (d, $J=2.0 \mathrm{~Hz}, 1 \mathrm{H}, \mathrm{H}-4), 6.22$ (d, $J=2.0 \mathrm{~Hz}$, $1 \mathrm{H}, \mathrm{H}-2) ;{ }^{13} \mathrm{C}$ NMR (DMSO- $\left.d_{6}, 75 \mathrm{MHz}\right) \delta$ : 178.9 (C-9), 166.7 (C-3), 163.2 (C-1), 157.7 (C-4a), 154.8 (C-4b), 138.4 (C-6), 127.6 (C-8), 121.9 (C-8a), 120.8 (C-7), 116.8 (C-5), 102.6 (C-8b), 98.8 (C-4), 94.7 (C-2); MS-ESI m/z: $309.1[\mathrm{M}+\mathrm{H}]^{+}$. 表征数据和理化性质与参考文献[32]一 致.

1,3-二羟基-8-甲氧基呫吨酮 (3i): 黄色粉末，产率 82\%. ${ }^{1} \mathrm{H}$ NMR (DMSO- $\left.d_{6}, 300 \mathrm{MHz}\right) \delta: 13.33$ (s, $1 \mathrm{H}$, OH-1), 7.70 (t, $J=8.4 \mathrm{~Hz}, 1 \mathrm{H}, \mathrm{H}-6), 7.06$ (d, $J=8.3 \mathrm{~Hz}$, 1H, H-7), 6.96 (d, $J=8.3 \mathrm{~Hz}, 1 \mathrm{H}, \mathrm{H}-5), 6.27$ (d, $J=1.8$ Hz, 1H, H-4), 6.12 (d, J=1.9 Hz, 1H, H-2), 3.89 (s, 3H, $\left.\mathrm{CH}_{3}\right) ;{ }^{13} \mathrm{C}$ NMR (DMSO- $\left.d_{6}, 75 \mathrm{MHz}\right) \delta: 180.5$ (C-9), 166.2 (C-8), 163.5 (C-3), 160.6 (C-1), 157.6 (C-4a), 156.8 (C-4b), 136.3 (C-6), 110.4 (C-8a), 109.7 (C-5), 107.0 (C-7), 102.8 (C-8b), 98.6 (C-4), 93.8 (C-2), $56.7\left(\mathrm{OCH}_{3}\right)$; MS-ESI $m / z: 259.2[\mathrm{M}+\mathrm{H}]^{+}$. 表征数据和理化性质与参 考文献[34]一致.

1,3-二羟基-[6,7]-苯骈呫吨酮(3j): 亮黄色粉末，产 率 84\%. ${ }^{1} \mathrm{H}$ NMR (DMSO-d $\left.6,300 \mathrm{MHz}\right) \delta: 12.90(\mathrm{~s}, 1 \mathrm{H}$, OH-1), 11.22 (s, 1H, OH-3), 8.83 (s, 1H, C-8), 8.23 (d, $J=$ $8.3 \mathrm{~Hz}, 1 \mathrm{H}, \mathrm{H}-5), 8.04$ (d, $J=7.1 \mathrm{~Hz}, 2 \mathrm{H}, \mathrm{H}-5, \mathrm{H}-8), 7.70$ (t, $J=7.1 \mathrm{~Hz}, 1 \mathrm{H}, \mathrm{H}-6), 7.57$ (t, $J=7.5 \mathrm{~Hz}, 1 \mathrm{H}, \mathrm{H}-7), 6.41$ (d, $J=2.1 \mathrm{~Hz}, 1 \mathrm{H}, \mathrm{H}-4), 6.21$ (d, $J=2.1 \mathrm{~Hz}, 1 \mathrm{H}, \mathrm{H}-2) ;{ }^{13} \mathrm{C}$ NMR (DMSO- $d_{6}, 300$ MHz) $\delta$ : 180.8 (C-9), 167.0 (C-3), 
163.8 (C-1), 158.3 (C-4a), 151.7 (C-4b), 136.8 (C-6), 130.1 (C-8), 129.9 (C-7), 129.6 (C-8), 127.6 (C-6), 127.6 (C-5), 126.3 (C-8a), 120.2 (C-7), 113.6 (C-5), 102.2 (C-8b), 98.2 (C-4), 94.8 (C-2); MS-ESI m/z: 279.3 [M+ $\mathrm{H}]^{+}$. 表征数据和理化性质与参考文献[35]一致.

1,3-二羟基-[5,6]-苯骈呫吨酮 (3k): 暗黄色粉末, 产 率 81\%. ${ }^{1} \mathrm{H}$ NMR (DMSO- $\left.d_{6}, 300 \mathrm{MHz}\right) \delta$ : $12.84(\mathrm{~s}, 1 \mathrm{H}$, OH-1), 8.57 (d, $J=7.4 \mathrm{~Hz}, 1 \mathrm{H}, \mathrm{H}-5), 8.06$ (d, $J=7.4 \mathrm{~Hz}$, $1 \mathrm{H}, \mathrm{H}-8), 8.00$ (d, $J=8.6 \mathrm{~Hz}, 1 \mathrm{H}, \mathrm{H}-8), 7.85$ (d, $J=9.0$ $\mathrm{Hz}, 1 \mathrm{H}, \mathrm{H}-7), 7.80 \sim 7.73$ (m, 2H, 1H, H-6), 6.61 (s, $1 \mathrm{H}$, $\mathrm{H}-4), 6.25$ (s, $1 \mathrm{H}, \mathrm{H}-2) ;{ }^{13} \mathrm{C}$ NMR (DMSO- $d_{6}, 300 \mathrm{MHz}$ ) $\delta: 179.9$ (C-9), 166.4 (C-3), 162.9 (C-1), 157.5 (C-4a), 153.3 (C-4b), 136.5 (C-6), 130.5 (C-7), 129.1 (C-12), 128.7 (C-5), 127.9 (C-11), 127.6 (C-13), 123.3 (C-8), 122.9 (C-10), 120.2 (C-7), 115.9 (C-8a), 103.0 (C-8b), 99.1 (C-4), 94.9 (C-2); MS (ESI) m/z: $279.3[\mathrm{M}+\mathrm{H}]^{+}$. 表 征数据和理化性质与参考文献[35]一致.

1,3-二羟基-7-乙酰氨基呫吨酮(31): 黄色粉末，产 率 78\%. UV-Vis $\left(\mathrm{CH}_{3} \mathrm{COCH}_{3}\right) \lambda_{\text {max }}: 336 \mathrm{~nm} ;{ }^{1} \mathrm{H}$ NMR (DMSO- $\left.d_{6}, 300 \mathrm{MHz}\right) \delta: 12.85(\mathrm{~s}, 1 \mathrm{H}, \mathrm{OH}-1), 11.10(\mathrm{~s}$, $1 \mathrm{H}, \mathrm{NH}), 10.27$ (s, 1H, OH-3), 8.41 (s, 1H, H-8), 7.95 (d, $J=8.0 \mathrm{~Hz}, 1 \mathrm{H}, \mathrm{H}-6), 7.55$ (d, $J=8.9 \mathrm{~Hz}, 1 \mathrm{H}, \mathrm{H}-5), 6.38$ (s, $1 \mathrm{H}, \mathrm{H}-4), 6.21$ (s, 1H, H-2), 2.09 (s, 3H, $\left.\mathrm{CH}_{3}\right) ;{ }^{13} \mathrm{C} \mathrm{NMR}$ (DMSO- $\left.d_{6}, 75 \mathrm{MHz}\right) \delta: 180.2$ (C-9), 167.0 (NHCO), 166.3 (C-3), 163.3 (C-1), 157.8 (C-4a), 151.6 (C-4b), 136.3 (C-7), 127.4 (C-8a), 120.2 (C-6), 118.6 (C-8), 114.0 (C-5), 102.5 (C-8b), 98.5 (C-4), 94.4 (C-2), $24.4\left(\mathrm{CH}_{3}\right)$; IR (KBr) $v: 3467,1639,1615,1523,1320,1184,832 \mathrm{~cm}^{-1}$; MS-ESI $m / z: 286.2[\mathrm{M}+\mathrm{H}]^{+}$; HRMS calcd for $\mathrm{C}_{15} \mathrm{H}_{12} \mathrm{NO}_{5}$ $[\mathrm{M}+\mathrm{H}]^{+}$286.0716, found 286.0715.

\subsection{2 清除 $\mathrm{NO}_{2}^{-}$作用实验}

\subsubsection{1 溶液的配置}

0.4\%对氨基苯磺酸溶液的配制: 称取 $0.4 \mathrm{~g}$ 对氨基 苯磺酸溶于 $100 \mathrm{~mL} 20 \%$ 盐酸中, 混合均匀, 避光保存.

$0.2 \% N-1$ - 荎乙二胺盐酸盐溶液的配制: 称取 $0.2 \mathrm{~g}$ 盐酸 N-1-荎乙二胺溶于 $100 \mathrm{~mL}$ 的蒸馏水混匀, 避光保 存.

亚硝酸钠标准溶液的配制: 准确称取在硅胶干燥器 中干燥了 $24 \mathrm{~h}$ 的亚硝酸钠 $0.1000 \mathrm{~g}$, 加水溶解, 定容至 $250 \mathrm{~mL}$ (浓度为 $400 \mu \mathrm{g} / \mathrm{mL}$ ), 作为储备液. 取储备液 $1.25 \mathrm{~mL}$ 稀释至 $100 \mathrm{~mL}$ (浓度为 $5 \mu \mathrm{g} / \mathrm{mL}$ ), 待用.

\subsubsection{2 实验方法}

原 $\mathrm{NO}_{2}^{-}$溶液的吸光度 ${ }^{[2,36]}$ : 取 $5 \mu \mathrm{g} / \mathrm{mL}$ 的 $\mathrm{NaNO}_{2}$ 标 准溶液 $3 \mathrm{~mL}$, 用磷酸氢二钠-柠檬酸缓冲溶液调节 $\mathrm{pH}$ 值等于 3 或 7, 室温下反应 $20 \mathrm{~min}$ 后, 立即加入 $2 \mathrm{~mL}$
$0.4 \%$ 对氨基苯磺酸溶液, 混匀, 静置 $3 \sim 5 \mathrm{~min}$ 后, 加 入 $1 \mathrm{~mL} 0.2 \%$ 盐酸 $N-1$ - 萗乙二胺溶液, 加水至刻度, 混 匀, 静置 $15 \mathrm{~min}$, 以 $5 \mathrm{~mL}$ 蒸馏水为空白, 在 $544 \mathrm{~nm}$ 处 测吸光度. 测得标准液的吸光度为 $A_{2}$.

加入待测样品后 $\mathrm{NO}_{2}^{-}$溶液的吸光度: 取 $3 \mathrm{mg} / \mathrm{mL}$ 的呫吨酮类化合物 $1 \mathrm{~mL}$ 于 $25 \mathrm{~mL}$ 容量瓶, 加入 5 $\mu \mathrm{g} / \mathrm{mL}$ 的 $\mathrm{NaNO}_{2}$ 标准溶液 $3 \mathrm{~mL}$, 用磷酸氢二钠-柠檬酸 缓冲溶液调节 $\mathrm{pH}$ 值等于 3 或 7, 室温下反应 $30 \mathrm{~min}$ 后, 立即加入 $2 \mathrm{~mL} 0.4 \%$ 对氨基苯磺酸溶液, 混匀, 静置 $3 \sim$ $5 \mathrm{~min}$ 后, 加入 $1 \mathrm{~mL} 0.2 \%$ 盐酸 $N-1$ - 荎乙二胺溶液, 加水 至刻度, 混匀, 静置 $15 \mathrm{~min}$, 以 $5 \mathrm{~mL}$ 蒸馏水为空白, 在 $544 \mathrm{~nm}$ 处测吸光度. 测定数据为 $A_{1}$.

亚硝酸根清除率的计算公式如下:

亚酸根的清除率 $=\frac{A_{2}-A_{1}}{A_{2}} \times 100 \%$

式中 $A_{2}$ 是原 $\mathrm{NO}_{2}^{-}$溶液的吸光度, $A_{1}$ 是加入待测样品后 $\mathrm{NO}_{2}^{-}$溶液的吸光度.

\section{References}

[1] Zhang, H.; Xu, G.; Yuan, J.-Y. J. Zhengzhou Grain College 2000, 21,50 (in Chinese). (张虹, 徐刚, 袁建耀, 郑州粮食学院学报, 2000, 21, 50.)

[2] Zhao, C.-H. Food Ind. 2010, 5, 4 (in Chinese). (薛长晖, 食品工业, 2010, 5, 4.)

[3] Zhu, Y.-X.; Feng, H.-J.; Sun, Y. Cancer 1994, 13, 470 (in Chinese). (褚衍信, 冯华进, 孙燕, 癌症, 1994, 13, 470.)

[4] Xiao, H.; Yang, L.; Zenai, C.; Guangming, L.; Jinfu, Q. J. Chin. Pharm. Sci. 2001, 10, 172.

[5] Ee, G. C. L.; Lim, C. K.; Lee, H. L. J. Asian Nat. Prod. Res. 2006, $8,567$.

[6] Wen, L.; Lin, Y.-C.; She, Z.-G.; Du, D.-S.; Chan, W.-L.; Zheng, Z. H. J. Asian Nat. Prod. Res. 2008, 10, 133.

[7] Nicole, P.; Panagiotis, M. Anti-Cancer Agents Med. Chem. 2009, 9, 77.

[8] Lesch, B.; Braese, S. Angew. Chem., Int. Ed. 2004, 43, 115.

[9] Sellés, A. J. N.; Castro, H. T. V.; Agüero, J.; González, J.; Naddeo, F.; De Simone, F.; Rastrelli, L. J. Agric. Food Chem. 2002, 50, 762.

[10] Luo, C.-T.; Mao, S.-S.; Liu, F.-L.; Yang, M.-X.; Chen, H.; Kurihara, H.; Li, Y. Fitoterapia 2013, 91, 140.

[11] Dube, M.; Zunker, K.; Neidhart, S.; Carle, R.; Steinhart, H.; Paschke, A. J. Agric. Food Chem. 2004, 52, 3938.

[12] Rukachaisirikul, V.; Kamkaew, M.; Sukavisit, D.; Taylor, W. C. J. Nat. Prod. 2003, 66, 1531.

[13] Park, H. H.; Park, Y.-D.; Han, J.-M.; Im, K.-R.; Lee, B. W.; Jeong, I. Y.; Jeong, T.- S.; Lee, W. S. Bioorg. Med. Chem. Lett. 2006, 16, 5580 .

[14] Zhou, T.; Ohkoshi, E.; Bastow, K. F.; Lee, K.-H. Bioorg. Med. Chem. Lett. 2012, 22, 3219.

[15] Masand, V. H.; Mahajan, D. T.; Patil, K. N.; Chinchkhede, K. D.; Jawarkar, R. D.; Hadda, T. B.; Alafeefy, A. A.; Shibi, I. G. Med Chem. Res. 2012, 21, 4523.

[16] Ichikib, H.; Okadab, M.; Ishidac, T.; Tanigawaa, K. Phytomedicine 2001, 8,85 .

[17] Zhang, X.-J.; Li, X.; Sun, H.-P.; Wang, X.-J.; Zhao, L.; Gao, Y.; Liu, X.-R.; Zhang, S.-L.; Wang, Y.-Y.; Yang, Y.-R.; Zeng, S.; Guo, 
Q.-L.; You, Q.-D. J. Med.Chem. 2013, 56, 276.

[18] Azevedo, C. M. G.; Afonso, C. M. M.; Sousa, D.; Lima, R. T.; Vasconcelos, M. H.; Pedro, M.; Barbosa, J.; Corrêa, A. G.; Reis, S.; Pinto, M. M. M. Bioorg. Med. Chem. 2013, 21, 2941.

[19] Peres, V.; Nagem, T. J.; De Oliveira, F. F. Phytochemistry 2000, $55,683$.

[20] Zou, H.; Koh, J.-J.; Li, J.-G.; Qiu, S.-X.; Aung, T. T.; Lin, H.-F.; Lakshminarayanan, R.; Dai, X.-P.; Tang, C.; Lim, F. H.; Zhou, L.; Tan, A. L.; Verma, C.; Tan, D. T. H.; Chan, H. S. O.; Saraswathi, P.; Cao, P.; Liu, S.-P.; Beuerman, R. W. J. Med. Chem. 2013, 56, 2359.

[21] Akrawi, O. A.; Mohammed, H. H.; Patonay, T.; Villinger, A.; Langer, P. Tetrahedron 2012, 68, 6298.

[22] Zou, Y.; Zhao, Q.-J.; Hu, H.-G.; Yu, S.-C.; Xu, M.-J.; Wu, Q.-Y. Arch. Pharm. Res. 2012, 35, 2093.

[23] Hu, L.-H.; Qin, Z.-L. Chin. J. Synth. Chem. 2002, 10, 285 (in Chinese).

(胡利红, 覃章兰, 合成化学, 2002, 10, 285.)

[24] Eaton, P. E.; Carlson, G. R.; Lee, J. T. J. Org. Chem. 1973, 38, 4071.

[25] Mehta, G.; Shah, S. R.; Venkataswarlu, Y. Tetrahedron 1994, 50,
11729

[26] Horne, S.; Rodrigo, R. J. Org. Chem. 1990, 55, 4520.

[27] Alix, J.; Jiang, H. Wardlaq, J. H. Aust. J. Chem. 1990, 43, 1745.

[28] Yen, C.-T.; Nakagawa-Goto, K.; Hwang, T.-L.; Morris-Natschke, S. L.; Bastow, K. F.; Wu, Y.-C.; Lee, K.-H. Bioorg. Med. Chem. Lett. 2012, 22, 4018.

[29] Lin, C.-N.; Chung, M.-I.; Liou, S.-J.; Lee, T.-H.; Wang, J.-P. J. Pharm. Pharmacol. 1996, 48, 532.

[30] Ando, H.; Hirai, H.; Fujii, M.; Hori, Y.; Fukumura, M.; Niiho, Y.; Nakajima, Y.; Shibata, T.; Toriizuka, K.; Ida, Y. J. Nat. Med. 2007, $61,269$.

[31] Das, A.; Shaikh, M. M.; Jana, S. Med. Chem. Res. 2013, 1.

[32] Zhou, T.; Shi, Q.; Chen, C-H.; Huang, L.; Ho, P.; Morris-Natschke, L. S.; Lee, K.-H. Eur. J. Med. Chem. 2012, 47, 86.

[33] Pillai, R. K. M.; Naiksatam, P.; Johnson, F.; Rajagopalan, R.; Watts, P. C.; Cricchio, R.; Borras, S. J. Org. Chem. 1986, 51, 717.

[34] Markham, K. R. Tetrahedron 1965, 21, 1449.

[35] Cho, H.-J.; Jung, M.-J.; Woo, S.; Kim, J.; Lee, E.-S.; Na, Y. Bioorg. Med. Chem. 2010, 18, 1010.

[36] Song, R.; Wei, R.-B. Food Res. Dev. 2007, 28, 25 (in Chinese). (宋茹, 韦荣编, 食品研究与开发, 2007, 28, 25.)

(Li, L.; Fan, Y.) 